\section{Military Technical College Kobry El-Kobbah, Cairo, Egypt}

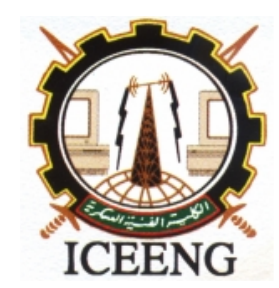

\section{$8^{\text {th }}$ International Conference on Electrical Engineering ICEENG 2012}

\title{
Analysis and classification of sleep EEG
}

\author{
By \\ Noha E. El-Kafrawy * \\ F. E. Z. Abou-Chadi ** \\ S. I. Rihan $* * *$
}

\section{$\underline{\text { Abstract: }}$}

In the present paper, a comparative study of performance for three techniques of feature extraction is presented in order to classify the sleep stages using EEG signals. A multilayer feed forward neural network was used for classification. Six sleep EEG records for each of ten patients were selected from Cairo Center of Sleep Disorder. Three methodologies of analysis were utilized for feature extraction. These include: autoregressive modeling (AR), bispectral analysis, and discrete wavelet transform (DWT), where principle component analysis (PCA) was used to reduce feature dimensionality. The features derived from the three methodologies of signal analysis were used as input feature vectors to the classifier. Information fusion is very important task in pattern recognition as it is difficult to develop classifiers with a high identification performance rate. The multilayer feed forward neural network gives higher classification rate using the data fusion at the feature extraction level. It reaches $83.4 \%$.

\section{Keywords:}

Autorgressive modeling, bispectral analysis, discrete wavelet transform, principle componenet analysis, multilayer feed forward neural network.

* Egyptian Armed Forces

** Benha High Technology Institute, Benha, Egypt

*** College of Engineering, Cairo University, Cairo, Egypt 


\section{Introduction:}

The use of Electroencephalogram (EEG) analysis in the study of sleep and sleep disorder is an ongoing research topic of vital importance to clinicians from wide medical interests [1]. Modern signal processing and pattern recognition techniques were combined in order to characterize important features of sleep EEG signals. Six sleep EEG records for each of ten patients were selected from Cairo Center of Sleep Disorder. Researchers have developed many techniques to analyze and classify the sleep stages using EEG records. Mendez et al. [2] found that the time-invariant and time-variant parametric models resulted fine tools for diagnosing the Obstructive sleep apnea (OSA)) . Satoshi Hagihiraet at al. [3] confirmed the methodology of bispectral analysis of EEG, which analyzes EEG waveform and calculates bispectrum, bicoherence, and power spectrum of EEG. T.Ning et al [4] investigated the EEG of the rat during various vigilance states to study the degree of Gaussianity of their amplitude distribution and to detect the presence of quadratic phase coupling. Moreover, several important characteristic waves in (EEGs) were reported using Daubechies wavelet by Min Soo Kim et al. [5]. Edgar Oropesa1 et al. [6] used a wavelet packet transformation to provide localized time-frequency information, and they used an artificial neural network for doing optimal classification. The classification results compared to those of a human expert reached 70 to $80 \%$ of agreement. Sheng Liu et al. [7] proposed a robust system that combines multiple signal-processing methods in a multistage scheme: integrated adaptive filtering, wavelet transform and artificial neural network. The detection rate of epileptic events was $90.0 \%$. Brijil Chambayil [8] used an Artificial Neural Network (ANN) training to detect the eye blink artificts. The performance of the Cascadeforward Back propagation (CFBP) network is better compared to the Feed-forward Back propagation (FFBP) networks in classifying a signal to an eye blink or not.

The present work aims to compare the performance of three methodologies of feature extraction in order to obtain the highest classification rate. These include: autoregressive modeling (AR), bispectral analysis, and discrete wavelet transform (DWT), where principal component analysis (PCA) was used to reduce feature dimensionality. The features derived from the three methodologies of signal analysis were used as input feature vectors to a neural network classifier. A multilayer feed forward neural network was used for classification. A data fusion technique was also used to improve the classification rate. The results have shown that the significant differences for the six sleep stages using different feature extraction techniques.

\section{Data Set:}

Ten patients aged between (35-50) years were selected from the Cairo center for sleep disorder to record their sleep EEG signals. EEG signals were recorded bilaterally using 
SAHC (sleep analyzer hybrid microcomputer system) from 6 p.m to about 3 a.m. The recordings were made when patient was laying down and relaxed in a quiet room with dim light, where the patient head was fixed in a stereotaxic frame connected with ballshaped silver electrodes with shielded cable for recording. The standard arrangement of the 10-20 system of electrodes was positioned on the human skull over the central and occipital lobes for the right and left sides. The ear or mastoid were used as recommended references for the electrodes measuring EEG to maximizes inter electrode distance and to avoid mixing activity from two different scalp areas. The sampling rate was $100 \mathrm{~Hz}$.

\section{Feature Extraction:}

\subsection{Autoregressive Modelling (AR):}

AR modelling proceeds by a series of well-defined steps. The first step: is to examine the stationarity of sleep EEG signals to determine if there was any trend to present in the data, such as increasing or decreasing amplitudes. The stationarity of the mean was tested using "Run test"[9]. It has been found that data are non stationary. Best fit curve of variable order ranging from 2 to 4 were applied to remove non-stationary of the sleep EEGs.

The second step: is to estimate the coefficients of the model. Coefficients of AR models can be estimated by Autocorrelation method.

The third step: is to select the model order. The model order selection using Yule Walker method for the six sleep stages. It has been found that the optimal model order which gives minimum ratio between residual power and original signal power is 6 .

The fourth step: is to test the model. A Kolmogrov-sminrov one sample test (K-S) test was used to test the randomness of the residual signal [10].

\section{$\underline{2.2 \text { Bispectral Analysis }}$}

For a zero-mean, stationary process $\{X(k)\}$, the third-order cumulant is defined as the expected value of the triple product [4]

$$
R(m, n)=E\{x(k) x(k+m) x(k+n)\}
$$

and the bispectrum is defined as the Fourier transform of the third-order cumulant[TOC] Sequence, i.e. 


$$
B\left(w_{1}, w_{2}\right)=\sum_{m=-\infty}^{\infty} \sum_{n=-\infty}^{\infty} R(m, n) e^{-j\left(w_{1} m+w_{2} n\right)}
$$

If the process $\{X(k)\}$ is truly Gaussian, then its third cumulants $R(m . n)=0$ for all (m. $\mathrm{n})$ and its bispectrum $B(w 1, w 2)=0$ for all $(w I, w 2)$. Consequently, a random process exhibiting a highly nonzero bispectrum can be considered as highly nonGaussian. Another useful property of the bispectrum we have utilized here is its ability to detect the quadratic phase coupling

$$
D=\sum_{\left(w_{1}, w_{2}\right)}\left|B\left(w_{1}, w_{2}\right)\right|
$$

To quantify the degree of a quadratic phase coupling, the bicoherence index is computed. This index is a function of the bispectrum $B(w 1, w 2)$ and the power spectrum $P(w)$ and is defined as:

$$
b i\left(w_{1}, w_{2}\right)=\frac{\left|B\left(w_{1}, w_{2}\right)\right|^{2}}{p\left(w_{1}\right) p\left(w_{2}\right) p\left(w_{1}+w_{2}\right)}
$$

The last equation denote that the bicoherence index measures proportion of a specified of energy in each pair of frequencies $w 1$ and $w 2$ components of a specified sample of the time series and their sum $w 3$ that satisfies the definition of quadratic phase coupling, i.e. the phase of $w 3$ must equal the phase of $w 1$ and $w 2$.

Since bicoherence is the proportion of the coupled to the uncoupled energy, it is normalized (by the product of the $w 1$ power $* w 2$ power*w3 power) and it is independent of the total energy [4].

The bicoherence index should have significance level exceeds 0.15 to indicate the existence of phase coupling between the harmonic components of the process [4].

In general, it is apparent that the bispectrum involves the phase information that the conventional power spectrum does not involve.

\section{$\underline{\text { 2.3 Discrete Wavelet Transform }}$}

A discrete wavelet decomposition was performed for signals of length 4096 from sleep EEG signal for the six sleep stages to the tenth level of resolution using "haar" 
mother function [12]. For each of the 11 resolution and approximation coefficients, the average energy content of the coefficients at each resolution was computed. There were a total of 9 sub bands from which features were extracted. The ith element of a feature vector was given [12]

$$
v i d u t=\frac{1}{n i} \sum_{j=1}^{n i} w_{i, j}^{2}
$$

$$
i=1,2 \ldots 12
$$

Where $n_{1}=2^{11}, n 2 \equiv 2^{8}, n 3 \equiv 2^{7}, \ldots, n_{12}=2^{0}, v_{i}^{d w t}$ is the $\mathrm{i}$ th feature element in a DWT feature vector; ${ }^{n} i$ is the number of samples individual sub band; and $n_{i, j}^{2} j_{\text {is }}$ the $j$ th coefficient of the $i$ th sub band. As a result, a DWT feature vector is formed as given by [13]

$$
v^{d w t}=\left\{v 1^{d w t}, v 2^{d w t}, \ldots v 1 \mathcal{O}^{d w t}\right\}
$$

Principal component analysis (PCA) is one of the most important recent methods of dimensionally reduction, where the representation of the input vector is in terms of eigenvectors is of their covariance matrix [15].

PCA has been applied to DWT coefficient matrices. This reduces the number of features to the first ten principal component of DWT.

\section{3. design of classifier:}

In order to classify the different stages of the sleep EEG signal a multi-layer feed forward neural network classifier was used [16].

The training and testing patterns were derived from 15 records for each of the 10 patient and using three feature vectors are: spectral analysis using AR modeling, the bispectral analysis, and DWT using PCA, separately. The designed neural network was trained and tested using the hold-out method [16] where 50\% of the records were used in the training phase and $50 \%$ were used in the testing phase.

The feature vectors used for training and testing the designed networks consisted of a 6 features vector derived from AR modeling features, a 2 features vector derived from bispectral analysis and a 10 features vector derived from wavelet analysis. The number of input neurons used is varied corresponding to the technique is using, the number of output neurons is fixed to 3 neurons, for classify each stage, and the number of the 
hidden neurons is obtained after a study of several configurations, during the training process.

Fusion at the feature extraction level where feature extracted using multiple techniques is concatenated. It combines feature vectors at The Feature Extraction Level the representation level to provide higher dimensional data points [17].

The percentage of correct classification of the feed forward multi-layer neural network as shown in Table 1.

Table (1): Percentage e of correct classification using multi-layer feed forward neural network for four feature sets.

\begin{tabular}{|c|c|c|c|c|}
\hline \multirow[t]{2}{*}{ Sleep stages } & \multicolumn{4}{|c|}{ \% Correct Classification } \\
\hline & $\begin{array}{c}\text { AR } \\
\text { coefficients }\end{array}$ & $\begin{array}{l}\text { Bispectral analysis } \\
\text { features }\end{array}$ & $\begin{array}{c}\text { DWT using } \\
\text { PCA }\end{array}$ & $\begin{array}{l}\text { Using data } \\
\text { fusion }\end{array}$ \\
\hline Stage wake & $80 \%$ & $73.3 \%$ & $71.3 \%$ & $83.3 \%$ \\
\hline Stage 1 & $78.6 \%$ & $70 \%$ & $56.6 \%$ & $81.3 \%$ \\
\hline Stage 2 & $83.3 \%$ & $66.6 \%$ & $74 \%$ & $84.6 \%$ \\
\hline Stage 3 & $73.3 \%$ & $58.6 \%$ & $68 \%$ & $76.6 \%$ \\
\hline Stage 4 & $86.6 \%$ & $60 \%$ & $76.6 \%$ & $88 \%$ \\
\hline Stage REM & $84 \%$ & $63.3 \%$ & $80 \%$ & $86.6 \%$ \\
\hline All stages & $80.8 \%$ & $65.3 \%$ & $71 \%$ & $83.4 \%$ \\
\hline
\end{tabular}

\section{Conclusions:}

In this work, the performance of three techniques of feature extraction was compared for the classification of sleep EEG. A multilayer feed forward neural network was used.

It has been concluded that using a data fusion technique for the three techniques of analysis together as input to the multilayer feed forward neural network gives more better results than using each technique of analysis separately as input to the multilayer feed forward neural network .

The results of classification have shown the significant differences for the six sleep stages using different analysis techniques. The multilayer feed forward neural network gives higher classification rate using the data fusion at the feature extraction level.

\section{References:}


[1] M.Akay. Biomedical signal processing. Academic Press, Inc., 1994.

[2] Mendez M.O., Villantieri O. P., Bianchi A. M., and Cerutti S. "Sleep Analysis for Wearable Devices Applying Autoregressive Parametric Models". Politecnico di Milano, Italia Proceedings of the 2005.

[3] Satoshi Hagihira, , Masaki Takashina, , Takahiko Mori, Takashi Mashimo, and Ikuto Yoshiya. "Practical Issues in Bispectral Analysis of Electroencephalographic Signals" Technical Communication, June 15, 2001.

[4] T.Ning and J.Bronzino,"Bispectral analysis of the rat EEG during Various Vigilance States". IEEE.Trans.Biomed.Eng, vol.36, no.4, 1989.

[5] Min Soo Kim, Young Chang Cho, Abibullaev Berdakh and Hee Don Seo. "Analysis of Brain Function and Classification of Sleep Stage EEG using Daubechies Wavelet". Sensors and Materials, Vol. 20, No. 1 (2008).

[6] Edgar Oropesa, Hans L. Cycon , Marc Jobert. " Sleep Stage Classification using Wavelet Transform and Neural Network". International Computer Science Institute, March 30, 1999

[7] He Sheng Liu_, Tong Zhang, and Fu Sheng Yang "A Multistage, Multimethod Approach for Automatic Detection and Classification of Epileptiform EEG" IEEE Transaction on Biomedical Engineering140, VOL. 49, NO. 12, DECEMBER 2002

[8] Brijil Chambayil, Rajesh Singla, R. Jha"EEG Eye Blink Classification Using NeuralNetwork"-Proceedings of the World Congress on Engineering 2010 Vol I WCE 2010, June 30 - July 2, 2010, London, U.K.

[9] J.S.M. Bendant and piersol. Random data: analysis and measurement procedures. New York: Willy, 1985.

[10] S. Seigel. Non-parametric statistics for behavioral sciences. Mcgraw-Hill, 1956.

[11] D.R. Brillinger," some basic aspects and uses of higher orde spectra," IEEE sig. Proc., vol.36, pp.239-249, 1994.

[12] Stephane Mallat. A Wavelet Tour of Signal Processing. Paris, Courant institute, New York University.

[13] R.M. Rao and A.S.bbopardikar. Wavelet Transforms: Introduction to theory, algorithms and applications. Wesely Longman, Inc., pp.1-30, 1998.

[14] Pablo Faundez Hoffman, Alvaro Fuentz, Sigitas Dagilis. K-complex detection using continuous wavelet transforms. Department of control Engineering. AAlborg University, December.2000.

[15] R. Schukoff. Pattern Recognition: Statistical, Structural, and Neural Approaches". John Wiley and Sons, Noy, 1992.

[16] J. Hwang, S. Kung, and M. Niranjam," the past, present and future of neural network for signal processing" IEEE Proc, Vol.14, no.6, 1997.

[17] Arun Ross and Anil Jain. Score normalization In Multimodal Biometrics System. Michigan State University, East Lansing. 21 october 2004. 
Proceedings of the $\boldsymbol{8}^{\text {th }}$ ICEENG Conference, 29-31 May, 2012 